\title{
Der Mensch im Mittelpunkt der Fabrik von morgen
}

\author{
Alexander Richter · Peter Heinrich · Alexander Stocker • Wolfgang Unzeitig
}

Eingegangen: 24. Mai 2015 / Angenommen: 8. August 2015 / Online publiziert: 29. August 2015

(C) Springer Fachmedien Wiesbaden 2015

Zusammenfassung Ungeachtet der wichtigen Impulse des Industrie 4.0-Begriffs für die Förderlandschaft im deutschsprachigen Raum, macht seine Unschärfe es Praktikern und Forschern schwer ihn einzuordnen, um konkrete Anwendungsfälle für Innovationsprojekte zu identifizieren. Hier setzt der vorliegende Beitrag an. Eine systematische Analyse von 35 großen, themenzugehörigen EU-Forschungsprojekten trägt zunächst zu einer besseren thematischen Orientierung bei. Im nächsten Schritt wird ein aktuell laufendes EU-Projekt vorgestellt, das sich mit der Entwicklung von Informations- und Kommunikationstechnologien (IKT) für Produktionsarbeiter der Zukunft auseinandersetzt. Drei aus dem Projektkontext gewählte Fallbeispiele illustrieren, wie IKT sich in Industriebetrieben einsetzen lassen, um den Menschen in den Mittelpunkt der Fabrik von morgen zu rücken. Am Beispiel der Dimension des befähigten Mitarbeiters, lässt sich zudem zeigen wie ein Produktionsarbeiter der Fabrik von morgen durch neue IKT insbesondere 1) Autonomie, 2) Kompetenz, 3) Verbundenheit und 4) Abwechslungsreichtum gewinnen kann. So ermöglicht der Beitrag einerseits die Orientierung an konkreten Anwendungsfällen und deren Reflexion sowie andererseits die Entwicklung eines ganzheitlichen Verständnisses für die sich derzeit und in den kommenden Jahren vollziehenden Veränderungen in vielen Industrieunternehmen.

Schlüsselwörter Industrie 4.0 - Smart Factory · Internet · Internet der Dinge · Internet of Everything

\section{A. Richter $(\bowtie) \cdot$ P. Heinrich Universität Zürich, Binzmühlestrasse 14, 8050 Zürich, Schweiz E-Mail: arichter@ifi.uzh.ch}

\author{
A. Stocker - W. Unzeitig \\ Virtual Vehicle Research Center, \\ Inffeldgasse 21/a, \\ 8010 Graz, Österreich
}


Abstract Notwithstanding the important push of the term "Industrie 4.0" towards research funding agencies in German-speaking countries, its fuzziness makes it challenging for researchers and practitioners to identify concrete application scenarios for innovation projects. The paper at hand enters the debate at this point: First of all, a systematic analysis of 35 big related EU research projects contributes to a better thematic orientation. Next, we introduce a current EU project looking into research and development of information and communication technology (ICT) for the production worker of the future. Three case studies from this EU project illustrate what kind of ICT can be implemented by industrial enterprises to put the human worker into the heart of future factories. The dimension of the "empowered worker" serves us to show how workers at factories of the future gain (1) autonomy, (2) competency, (3) connectedness and (4) work variety trough newly introduced ICT. Thus, the paper at hand facilitates the orientation towards concrete use cases and their reflection, and at the same time it facilitates the development of a comprehensive understanding for current and future transformations in many industrial enterprises.

Keywords Industrie $4.0 \cdot$ Smart factory $\cdot$ Internet $\cdot$ Internet of things $\cdot$ Internet of everything

\section{Der Faktor Mensch in der Industrie 4.0}

Der Begriff ,Industrie 4.0“ steht für die vierte industrielle Revolution und ihren Auslöser - das Internet und seine zahlreichen Facetten wie Daten, Dienste und Dinge (Kagermann et al. 2013). Dabei steht das Internet nur stellvertretend für technologische Weiterentwicklungen wie Smartphones oder RFID. Als eingebettete Systeme sorgen diese dafür, dass Produkte und Maschinen selbstständig Informationen untereinander austauschen können. Der industrielle Prozess wird zunehmend nicht mehr zentral aus der Fabrik heraus organisiert, sondern dezentral und dynamisch gesteuert. Das vierte industrielle Zeitalter ist somit durch eine zunehmende „Informatisierung“ nicht nur einzelner Fabriken, sondern ganzer Produktions-Wertschöpfungsnetzwerke charakterisiert.

Durch den Einzug von Informations- und Kommunikationstechnologien in physische Produkte sowie in ihre Produktionsstätten werden Maschinen intelligenter und vernetzter: Aus mechatronischen Produkten entstehen vernetzte cyber-physikalische Systeme (CPS), und klassische Produktionssysteme transformieren zu cyber-physikalischen Produktionssystemen (CPPS) (Denger et al. 2014). Beide bedienen sich einer Vielzahl unterschiedlicher Sensoren, um Daten aus der physischen Welt zu verarbeiten und Aktoren, um Vorgänge in der physischen Welt auszulösen. Als smart, connected products bezeichnet, sind sie im Begriff, Geschäftsmodelle von Unternehmen und ganzen Branchen zu verändern (Porter und Heppelmann 2014). Diese technologischen und wirtschaftlichen Veränderungen werden von einer steigenden Anzahl an Initiativen und Studien untersucht und gewinnen auf europäischer Ebene durch Begriffe wie „Factory of the Future (FoF)“, „Smart Factory“ oder „Advanced Manufacturing" zunehmend an Bedeutung. 
Zusammen mit den technologischen Aspekten stellt sich jedoch auch die Frage nach der zukünftigen Rolle des Menschen im Produktionsumfeld. In diesem Zusammenhang weist etwa die „Factories of the Future PPP Roadmap“ die Bedeutung des Wissensarbeiters als Schlüsselressource für die industrielle Wettbewerbsfähigkeit (vgl. EFFRA 2013) hin. Die „European Factory of the Future Research Association“ (EFFRA) hebt drei Kernaspekte hervor, um die zukünftige Rolle des Menschen und sein Arbeitsumfeld in Fabriken zu gestalten: 1) menschliches Arbeiten und Lernen, 2) Mensch-Maschine-Interaktion und 3) Mehrwert des Menschen für die Produktion. Eine weitere Studie von Fraunhofer IAO zur Produktionsarbeit der Zukunft (Spath et al. 2013), hebt die Rolle des Menschen in der Produktion hervor indem sie betont, dass Mensch-zentrierte IKT-Ansätze dringend zur Realität in produzierenden Betrieben werden müssen, um diese attraktiver für qualifizierte Mitarbeiter zu machen. Automatisierung wird laut dieser Studie für immer kleinere Serien möglich, und gerade deswegen bleibt menschliche Arbeit ein wichtiger Bestandteil der Produktion. Auch einer der Initiatoren des Industrie 4.0-Begriffs, Henning Kagermann, betont, dass nach einer Welle der Standardisierung und Automatisierung der nächste Schritt in der Fabrik der Zukunft darin bestehen wird, dass es immer weniger fest vorgeschriebene Arbeitsschritte geben wird und die Arbeitsinhalte häufiger wechseln werden (Kagermann 2014). In diesen zunehmend von Flexibilität geprägten Arbeitsbereichen spielen menschliche Produktionskräfte als Entscheider und Problemlöser eine wichtige Rolle.

Alle diese Akteure skizzieren also ein neues Rollenbild des Menschen in der Produktion, dessen Flexibilität und Entscheidungsverantwortung durch verbesserte IKT optimal unterstützt werden soll. Doch aufgrund der Unschärfe von Industrie 4.0 ist es für Unternehmen noch immer eine große Herausforderung, konkrete Anwendungsfälle für Innovationsprojekte zu identifizieren.

Vor diesem Hintergrund möchte der vorliegende Beitrag Praktikern und Forschern eine bessere Orientierung ermöglichen. Als Grundlage für eine verbesserte thematische Orientierung dient im Weiteren eine systematische Analyse von 31 großen, themenzugehörigen EU-Forschungsprojekten, die sich insbesondere mit Technologien zur Unterstützung des Menschen im Produktionsumfeld auseinandersetzen. Es zeigt sich, dass „der Mensch in der Fabrik der Zukunft" schon ein Thema war, dem zahlreiche durch die Europäische Kommission geförderte Projekte gewidmet wurden, bevor der Begriff „Industrie 4.0“ überhaupt aufkam. Im nächsten Schritt wird ein aktuell laufendes EU-Projekt vorgestellt, das sich mit der Entwicklung von Informations- und Kommunikationstechnologien für Produktionsarbeiter der Zukunft auseinandersetzt. Drei aus dem Projektkontext gewählte Fallbeispiele illustrieren, welche Technologien sich von Industriebetrieben einsetzen lassen, um den Menschen in den Mittelpunkt der Fabrik von morgen zu rücken. Anschließend wird insbesondere die Dimension des befähigten Mitarbeiters diskutiert. Dabei stellt die Self-Determination Theory (SDT) (Deci et al. 1989) ein hilfreiches Rahmenwerk dar, um die Lösungen auf ihre Befähigungspotentiale hin zu untersuchen.

\section{Initiativen auf europäischer Ebene}

Bereits seit 2008 fördert die Europäische Union Initiativen, die den Menschen im Mittelpunkt der Produktion von morgen sehen. Bis zum jetzigen Zeitpunkt finden 
sich 35 Projekte im Rahmen der Factories of the Future-Initiative, insbesondere in den definierten Domänen „Collaborative and Mobile Enterprises“ und „Human Centred Manufacturing", bei denen die Rolle des Menschen in der Produktion einen zentralen Forschungsschwerpunt darstellt ${ }^{1}$. Abbildung 1 fasst die zentralen Themenstellungen der einzelnen Projekte überblicksartig zusammen.

Etwa die Hälfte der Projekte beschäftigt sich mit dem Thema „kollaborative und mobile Unternehmen“. Die Projekte identifizieren Lücken und ungenutzte Potentiale bei der Nutzung von IKT in der produzierenden Industrie. Durch das Heben dieser Potentiale entstehen für Mitarbeiter und Anwender neuartige Werkzeuge, Methoden, Prozesse und Geschäftsmodelle (unter anderen CREMA, ROBUSTPLANET, PROSECO, ROAD4FAME).

In Unternehmensnetzwerken von Produktionsbetrieben mit komplexen Wertschöpfungs- und Lieferantenketten wird an Lösungen wie beispielsweise Cloudbasierte Web-Services, Everything-as-a-Service (XaaS) und selbst organisierende virtuelle Netzwerke für Geschäftstätigkeiten geforscht (unter anderen CREMA, IMAGINE, GLONET). Das Ziel dieser Projekte wird unterschiedlich definiert. Zum einen werden neue Methoden und Werkzeuge entwickelt bzw. weiterentwickelt, die die besonderen Anforderungen der Produktion sowie der Produktionsmitarbeiter berücksichtigen sollen. Zum anderen werden neue Geschäftsmodelle für flexible und agile Produktionsnetzwerke erforscht, die der Kunde sowohl als Einzelperson als auch als Unternehmen für besonders individualisierte Produkte auf möglichst einfache Art und Weise abrufen kann (unter anderen MEMAN, FLEXINET, USE-IT-WISELY). Die Begriffe „Cloud Manufacturing“ und „Global Production Network“ werden hierbei geprägt. Eine besondere Betrachtung erfahren dabei Unternehmensschnittstellen, die möglichst einfach gestaltet werden sollen. Zusätzlich sollen ökonomische Anforderungen wie Ressourcen-, Energieverbrauch und $\mathrm{CO}_{2}$-Produktion in einer Lebenszyklusbetrachtung mitberücksichtigt werden. Spezielle Fragestellungen finden sich auch bzgl. des Anwendungsfalls „kleine und mittlere Unternehmen“ (unter anderen MEMAN, ADVENTURE) sowie in der Integration der Mitarbeiter der Produktentwicklung (unter anderen EPES, IMAGINE). Die Schnittstelle Produktentwicklung, Fabrikplanung und Produktion bietet hierbei besondere Herausforderungen in der Abstimmung aufgrund auftretender Unsicherheiten (Unzeitig et al. 2014).

Neben den Potentialen in den Dienstleistungen durch IKT nehmen sich einige Projekte auch der Herausforderung an, digitale mobile Geräte für den Menschen als Anwender in die Produktion zu integrieren (beispielsweise EASY-IMP, COMVANTAGE). Auch ein Maßschneidern der Lösung für spezifische Rollen und für verschiedenste Akteuren in den Unternehmen, wie beispielsweise Produktionsarbeiter oder Manager, hat eine besondere Bedeutung. Hierzu liefert ein effizientes Zur-Verfügung-Stellen von Informationen mit entsprechender Zugänglichkeit einen wichtigen Beitrag. Gerade ein Teilen des angeeigneten Wissens mit anderen Mitarbeitern oder auch zwischen kooperierenden Unternehmen, individuelle Lernmöglichkeiten

\footnotetext{
${ }^{1}$ Die Projekte werden im Rahmen der Factories of the Future PPP im Bereich der angewandten Produktionsforschung gefördert und fanden im Zeitraum von 2008 bis 2013 im Rahmen des 7. Rahmenprogramms sowie seit 2014 im Rahmen des Horizon 2020 Rahmenprogramms statt. Siehe auch: (EFFRA 2013) und (European Commission 2015)
} 


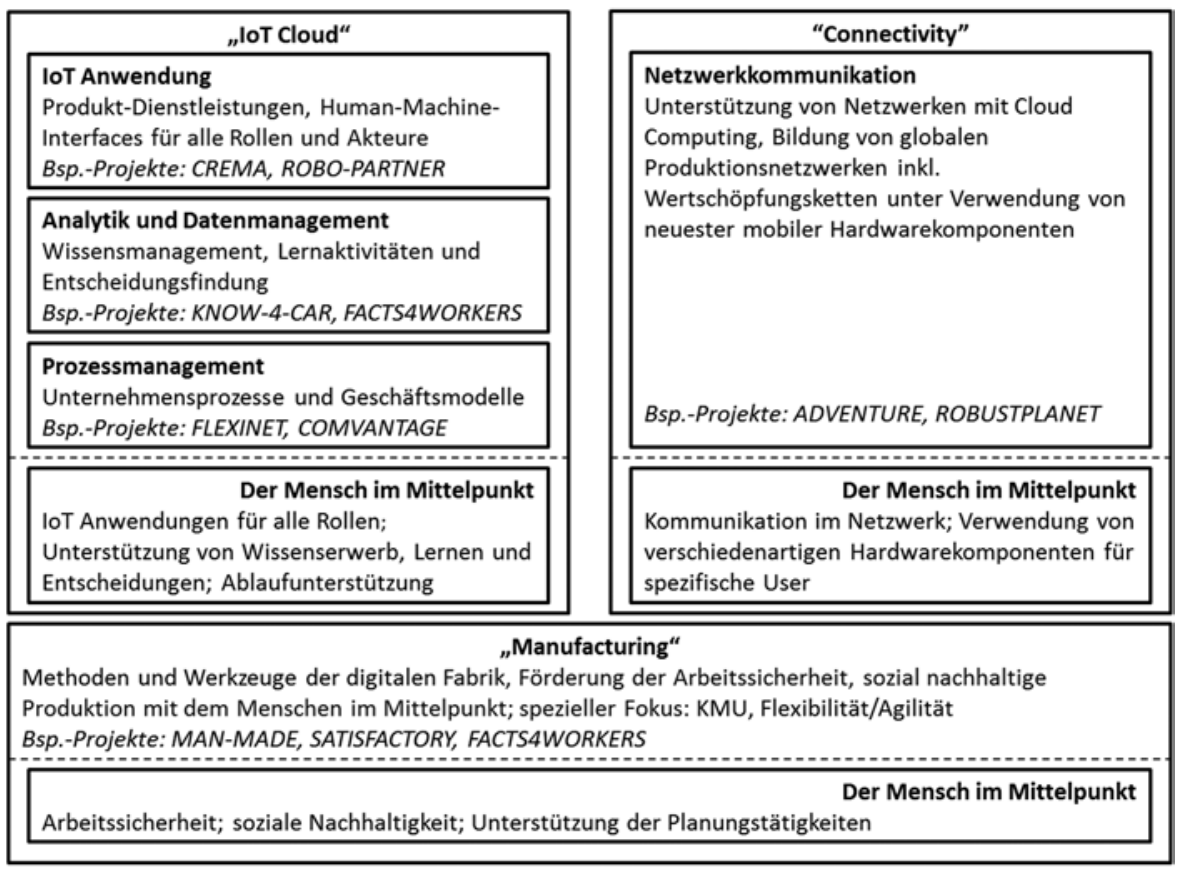

\begin{tabular}{l} 
"Engineering \\
Berücksichtigung von Produktentwicklungsthemen in der Produktion, Verbesserung der Schnittstelle \\
Engineering-Produktion, Nutzung von neuen Produktionstechnologien für neuartige Produkte \\
Bsp.-Projekte: MANSYS, EPES, IMAGINE \\
\hline Unterstützung der abteilungsübergreifenden Schnittstellen und Kommunikation sowie Wissenserwerb \\
\hline
\end{tabular}

Abb. 1 Überblick über die betroffenen Themengebiete der FoF-Projekte in den Domänen „Collaborative and Mobile Enterprises“ sowie „Human Centred Manufacturing“. (Auf Basis des IoT-Technology Stack (Wortmann und Flüchter 2015, nach Porter und Heppelmann 2014), ergänzt um Manufacturing und Engineering, mit Beispielprojekten. Es wurden die Zusammenfassungen der Projekte hinsichtlich der beschriebenen Tätigkeiten und Schlüsselwörter inhaltlich analysiert und auf Basis der Schlüsselwörter in übergeordnete Themengebiete gegliedert. Themen außerhalb von „Internet of Things“, in diesem Fall Produktion und Entwicklung, werden ebenfalls in eigenen Zellen angeführt. Die letzte Zeile je Themengebiet beinhaltet mehrere FoF-Beispielprojekte. Im Anhang befindet sich die gesamte Zuordnung der Projekte zu den Themengebieten zur detaillierten Information)

sowie Unterstützung bei Entscheidungen bieten ein umfangreiches Feld für Ideen und Weiterentwicklungen. Wieder werden in diesen Projekten bereits Kernbereiche von Industrie 4.0 behandelt, wobei die Kollaboration ausgehend von automatischer Kommunikation von Maschinen und unterstützter Kommunikation in Unternehmensnetzwerken um komplexe menschliche Aktivitäten wie Lernen und Wissenstransfer ergänzt werden.

Große Forschungsgebiete in diesen FoF-Projekten behandeln bereits Themen, die den Menschen in das Zentrum der Untersuchung stellen. Angefangen bei geeigneten und performanten Anwenderschnittstellen (unter anderem SO-PC-Pro, SO-SMART, SENSE\&REACT), über Systeme von Menschen und Maschinen, die miteinander 
interagieren (unter anderen LIAA, KNOW-4-CAR, SO SMART), bis hin zu einer Roboter-Mensch-Kollaboration (unter anderen ROBO-PARTNER, ROBO-MATE, FACTORY-IN-A-DAY), die ebenfalls die Arbeitssicherheit in höchstem Maß gewährleistet, wird an Fortschritten und Weiterentwicklungen hinsichtlich Schnittstellen zwischen Menschen und Maschinen gearbeitet. Hierbei ist die soziale Nachhaltigkeit in der Produktion von entscheidender Bedeutung.

\section{Vorgehen im Projekt}

Das Projekt FACTS 4WORKERS sieht das besondere Potential in der Nutzung von IKT darin, den Mitarbeiter zu stärken und ihm zur rechten Zeit, in geeigneter Weise Informationen zur Verfügung zu stellen, die ihm unter anderem als Entscheidungshilfe oder zur Lösungssuche bei Problemen dienen können. Vom Internet of Things, das vielfach die Definition von Industrie 4.0 prägt, bewegt sich das Forschungsgebiet wieder auf den Menschen zurück, der einerseits von den gefertigten Produkten als Verbraucher profitieren und andererseits eine optimale Arbeitsstätte in der Produktion vorfinden soll, um den Produktionsstandort Europa für die Zukunft weiterhin attraktiv zu halten.

So soll zur Vision einer so genannten „Smart Factory“ beigetragen werden, in der „Smart Workers“ bestmöglich durch Informations- und Kommunikationstechnologie unterstützt werden. Hierdurch soll dem Menschen als Wissensträger eine zentrale Funktion in der Produktion zukommen. In einer Smart Factory, der Fabrik der Zukunft, steht der Mensch als flexibelstes Element der Produktionsabläufe im Mittelpunkt der Aufmerksamkeit. Seine Rolle geht weit über die herkömmlichen, automatisierten Routinetätigkeiten der Fabrikarbeit hinaus. Als Wissensarbeiter wird er durch eine autonome Arbeitsumgebung unter anderem dabei unterstützt, selbst neue Möglichkeiten zur kontinuierlichen Verbesserung von Wissensaustausch am Arbeitsplatz zu entwickeln. Von zentraler Bedeutung ist somit, dass er sich einerseits effizient und effektiv neues Wissen aneignen kann, beispielsweise als Grundlage für eine verbesserte Entscheidungsfindung. Andererseits soll er in die Lage versetzt werden sein erworbenes Wissen und seine Erfahrungen an andere Mitarbeiter in geeigneter Weise zur Verfügung zu stellen.

Im Projekt stellt sich somit die Frage, wie Menschen arbeiten und lernen, wie sie mit neuen Technologien interagieren und wie sich für sie ein attraktiver und fordernder Arbeitsplatz gestalten lässt, der ihre Zufriedenheit und Arbeitsmotivation erhöht. Die Antworten auf diese Fragen sind der Schlüssel zu erfolgreichen soziotechnischen Lösungen in Produktionsprozessen. Hierdurch soll das Projekt FACTS 4WORKERS helfen, das Thema Industrie 4.0 mit besonderem Fokus auf den Produktionsarbeiter als Individuum zu definieren, Lösungen dafür bereitzustellen und zum vielfältigen Diskurs des Begriffs Industrie 4.0 beizutragen.

Im Fokus der Untersuchungen stehen die täglichen Routinen der Industrie-Arbeiter, die sich über die Zeit entwickelt haben. Diese individuellen Praktiken stehen den top-down spezifizierten Produktionsprozessen gegenüber und ermöglichen ein tieferes Verständnis für die individuellen Bedarfe (vgl. Abb. 2). Bei der Analyse kommen moderne Datenerhebungsmethoden zum Einsatz, die von den Forschungspartnern 
Abb. 2 Vorgehen im Projekt

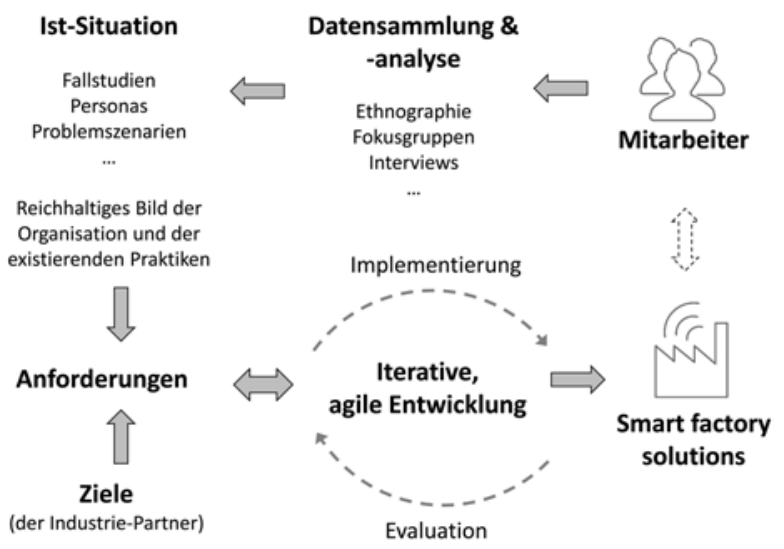

weiterentwickelt werden. Dabei werden unter anderem ethnographische Methoden und semi-strukturierte Interviews mit neueren Ansätzen wie Storytelling kombiniert. Daneben kommen auch neue Technologien wie Point-of-view-Kameras zum Einsatz.

Als Resultat werden in einem iterativen Prozess Anforderungen an eine Infrastruktur definiert und verfeinert, die Produktionsarbeiter befähigt (bessere Entscheidungsfähigkeit, erhöhte Teilhabe, erhöhte Autonomie) und schützt (reduziertes Stresslevel, reduzierte kognitive Überlastung, Reduktion monotoner, fehleranfälliger Arbeit). Obwohl es die festgeschriebene, oberste Maxime des Projektes ist, die Arbeitszufriedenheit nachhaltig zu erhöhen, werden die ausgewählten Anwendungsfälle auch die Produktionsqualität und -effizienz erhöhen (vgl. auch Abb. 3).

Die Daten für die folgenden Fallbeispiele wurden bei mehreren Aufenthalten bei den Industriepartnern erhoben. In den drei Unternehmen wurden bisher u. a. 22 Interviews und jeweils ein Fokusgruppen-Workshop durchgeführt.

\section{Fallbeispiele}

Wie einführend erläutert dienen die Fallbeispiele dazu, die Bandbreite möglicher Interventionen aufzuzeigen. Es werden sowohl bestehende Herausforderungen als auch Lösungsansätze gezeigt, um die identifizierten Probleme zu adressieren. Insbesondere ist zu erkennen, dass die bestehenden Unternehmensstrukturen einen großen Einfluss auf die Auswahl und den Einsatz der Technologien haben.

\subsection{Fallbeispiel 1: Awareness für flexible Produktionsmitarbeiter}

EMO-Orodjarna d.o.o. (EMO), ein seit mehr als 100 Jahren bestehendes mittelständisches, slowenisches Unternehmen, widmet sich der Herstellung von hoch-spezialisierten Werkzeugen für die Blechumformung und beliefert aktuell insbesondere die Automobilindustrie. In der Produktion sind die Stückzahlen identischer Werkzeuge sehr gering, in den meisten Fällen wird nur ein einziges Exemplar hergestellt. Die Komponenten dieser Werkzeuge werden vor Ort gefertigt und montiert. Auch wenn die Werkzeuge nach ähnlichen Prinzipien gestaltet werden, ist nahezu jedes Werk- 


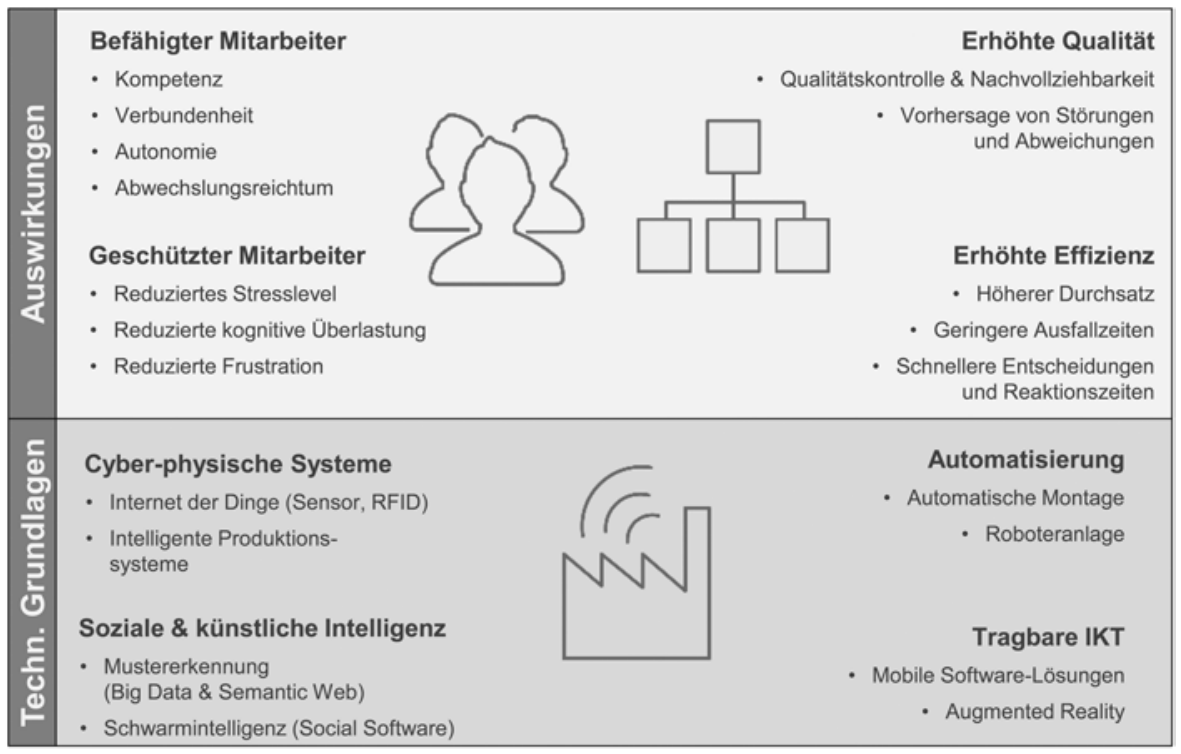

Abb. 3 Technische Grundlagen und Auswirkungen von Industrie 4.0

zeug einzigartig und seine Fabrikation verlangt damit ein hohes Maß an Agilität. Die Herstellung der Werkzeuge erfolgt parallel, Komponenten werden ,just-in-time“ gefertigt. Dadurch wird eine hohe Auslastung aller Ressourcen wie z. B. von Maschinen erreicht. Dies hat einerseits eine kontinuierliche Anpassung der eigenen Arbeitsprozesse zur Folge. Andererseits zeichnet sich die notwendige Agilität auch durch eine dezentrale und selbstorganisierende Steuerung des Herstellungs- und Montageprozesses ab. Dieser ist zwar grob vorgegeben, muss aber in den Details dynamisch von den Mitarbeitern moderiert werden. Dies geschieht in einer flachen Hierarchie meist durch direkte Kommunikation und Interaktion zwischen Mitarbeitern. Der starke Einbezug der Mitarbeiter in die Planungsprozesse bietet dabei den Vorteil, dass einerseits die emergenten Prozesse genau auf die Bedürfnisse der Mitarbeiter in der aktuellen Situation zugeschnitten werden können. Andererseits assoziieren sich Mitarbeiter aufgrund dieser Planungsaktivitäten auch verstärkt mit den Unternehmenszielen, da sie sich stets ein Gesamtbild über den Betrieb verschaffen müssen, um ihre Aktivitäten zu planen und zu priorisieren.

Herausforderung. Neben den Vorteilen einer dynamischen und „eingespielten“ Produktionssteuerung ergeben sich hierbei jedoch starke punktuelle Belastungen der Mitarbeiter, insbesondere wenn eine Situation die Betrachtung der Gesamtlage erfordert. Schnell bilden sich hierbei großflächige Kommunikations-Netze, da Informationen von vielen Stellen eingeholt werden müssen. Stehen beispielsweise benötigte Bauteile während der Montage nicht bereit, muss geklärt werden, in welchem Stadium der Fertigung sich diese Bauteile befinden und wann mit der Fertigstellung zu rechnen ist. In einigen Fällen können die Mitarbeiter aus der Produktion dann auch spontan Teile nachfertigen oder nachbearbeiten. Die gesamte Koordination 
diesbezüglich erfolgt ,auf Zuruf“. Folglich sind Entscheidungen einzelner Mitarbeiter wesentlich für die Einhaltung von Terminzusagen verantwortlich. Größere außerplanmäßige Arbeiten müssen aber mit dem Produktionsleiter abgeglichen werden. Die Mitarbeiter sind sich der daraus erwachsenen Verantwortung jedoch bewusst und nehmen diese auch mit großem Einsatz an.

Lösungsansatz. Ziel der Lösung ist die systematische Unterstützung der Mitarbeiter, bei gleichzeitigem Erhalt von Agilität, Autonomie und Flexibilität. Ein großes Potential bietet dabei die Vernetzung der Mitarbeiter mittels mobilen Endgeräten, um die Informationstransparenz zu erhöhen und damit die Koordination zu verbessern. Die Belastungen der Mitarbeiter entstehen in dem angeführten Fallbeispiel vor allem durch die unzureichende Informationslage über die Tätigkeiten anderer am Prozess beteiligter Mitarbeiter. Daher soll ein dezentrales, vernetztes System den Mitarbeitern auf einfache Weise erlauben zu kommunizieren, an welchen Teilen sie gerade arbeiteten oder auf welche Teile sie gerade warten. Somit bleibt die agile und selbstorganisierende Planung vollständig erhalten und die Mitarbeiter werden auch nicht in Ihrer Autonomie und Verantwortung beschnitten. Vielmehr ist jeder einzelne Mitarbeiter durch dieses System in die Lage versetzt sich in Echtzeit einen Gesamtüberblick über den Zustand der Produktion zu machen. Die Lösung erhöht die Zufriedenheit der Mitarbeiter, da Stress und zeitraubende Aktivitäten (wie zum Beispiel das Suchen von Teilen) reduziert werden. Ebenfalls vereinfacht die Lösung die eigene Planbarkeit der täglichen Aktivitäten, da auf Engpässe frühzeitig reagiert werden kann.

\subsection{Fallbeispiel 2: Selbstlernender Produktionsarbeitsplatz}

In dem weitaus größeren Unternehmen Hidria werden unter anderem Glühkerzen für Dieselmotoren hergestellt. Im Gegensatz zum ersten Fallbeispiel handelt es sich hierbei um Massenfertigung mit Kapazitäten jenseits von 100.000 Stück pro Woche und Fertigungslinie. Dies ist nur durch einen hohen Grad an Automatisierung zu erreichen, und somit erfolgt der Zusammenbau der Glühkerzen vollautomatisch. Menschliche Interaktion ist hierbei vor allem notwendig, um den Betrieb der Produktionsmaschinen aufrecht zu erhalten. Da die Fertigungslinien eine hohe technische Komplexität aufweisen, sind ein tiefgreifendes Verständnis ihrer Wirkungsweise und ein analytisches Vorgehen bei der Störungsbeseitigung unabdingbar, um eine hohe Verfügbarkeit der Maschinen zu gewährleisten. Die Mitarbeiter von Hidria arbeiten teils jahrelang im Schichtbetrieb mit den Maschinen, so dass ihr angeeignetes Erfahrungswissen einen elementaren Bestandteil der Wissens-Basis des Unternehmens darstellt, um einen reibungslosen Produktionsablauf zu gewährleiten.

Herausforderung. Im geschilderten Fall hängt die Effizienz der Produktionslinie insbesondere von der Problemlösungseffektivität einzelner Mitarbeiter ab. Diese möchten den Betrieb schnellstmöglich wieder aufzunehmen, um die Produktionsvorgaben zu erreichen. Zwar bringen die Problemlösungs-Episoden Abwechslung in die sonst eher monotonen Abläufe, werden aber nach einiger Zeit nicht mehr als Herausforderung gesehen, sondern als unvermeidliches Übel. Gleiches gilt für die Umstel- 
lung der Produktion auf ein anderes Produkt, beispielsweise ein anderes Modell von Glühkerzen. Besonders bei der Inbetriebnahme sind die Mitarbeiter gefordert, mit wenig Ausschuss und in kurzer Zeit wieder Glühkerzen zu produzieren, welche die geforderten Qualitätsanforderungen einhalten.

Lösungsansatz. Im Gegensatz zum ersten Fallbeispiel sind die Potentiale von ITLösungen eher in dem Zusammenspiel zwischen Produktionslinie und Mitarbeiter selbst zu suchen. Zur Lösung der beschriebenen Probleme kommt in diesem Fallbeispiel das Konzept eines selbstlernenden Montage-Arbeitsplatzes - zu verstehen als Mensch-Maschine-System - zum Einsatz. Insbesondere durch die Analyse von Anlagendaten (Entwicklung gemessener Toleranzen über die Zeit, aufgetretene Probleme, durchgeführte Wartungsarbeiten, ...) ist es möglich, Probleme zu detektieren, bevor sich diese durch Ausschuss oder die Abschaltung der Produktionslinie manifestieren. Die Maschine ,lernt' die bei der Produktion entstehenden Daten auf demnächst auftretende Probleme hin zu analysieren und diese frühzeitig vorherzusagen. Bei auftretenden Problemen gibt die Maschine dem Mitarbeiter eine Hilfestellung zur Lösungsfindung und unterstützt ihn bei seinen Entscheidungen zur Problemlösung. Wegen der hohen Komplexität dieser Maschinen scheint eine statische Datenbank mit Problemen und Lösungen als nicht zweckdienlich. Vielmehr kann diese Datenbank daher mit jedem weiteren aufgetretenen Problem auch um dessen Lösung erweitert werden. Dies dient zu einer Befähigung der Mitarbeiter, Probleme schneller zu lösen, ohne auf die Hilfe von Vorgesetzten oder des Wartungs-Teams angewiesen zu sein.

\subsection{Fallbeispiel 3: Kompetenzmanagement in der Qualitätssicherung}

Bei der dritten Fallstudie handelt es sich um ein Werk des weltweit tätigen Automobilzulieferers Schaeffler AG. Im Werk werden verschiedene Motorenelemente produziert. In den vergangen Jahren hat das Werk die Produktion von einer Fertigung, die nach Werkstattbereichen getrennt ist, hin zu einer modernen Wertstromfertigung umgestellt. Dies bedingte auch eine Neugestaltung des bestehenden Qualitätsmanagements und der hier näher betrachteten, operativen Qualitätssicherung (QS) und brachte mehrere Änderungen mit sich:

Zunächst einmal ist ein Mitarbeiter in der operativen QS seit der Umstellung nicht mehr nur für die Qualitätssicherung eines Teilbereichs der Fertigung zuständig, sondern für die gesamten Fertigungsschritte eines Wertstroms und für alle damit verbundenen Technologien. Dies hat einen höheren Anspruch an das ohnehin bereits umfassende Qualifikationsprofil eines QS-Mitarbeiters zur Folge: Aufgrund der Umstellung benötigt er nun Expertise über eine weitaus größere Anzahl an Typen von Produktionsmaschinen und zugehörigen Messeinrichtungen.

Des Weiteren bedeutet die Orientierung am Wertstrom, dass ein Produktionsbereich für die QS verantwortlich ist. Folglich ist ein QS-Fachbereich organisatorisch einem Produktionsbereich unterstellt und agiert in dessen Rahmen als Dienstleister. Als solcher unterstützt der QS-Fachbereich jeden Produktionsmitarbeiter in der täglichen Problemlösung z. B. bei der Behebung einer Störung einer Messstation. Daneben unterstützt der QS-Fachbereich bei Neuanläufen von Produkt-Typen, indem 
beispielsweise neue Einstellungen an Maschinen zeitnah geprüft und abgenommen werden. Nicht zu vergessen sind weitere regelmäßige Aufgaben mit denen ein QSMitarbeiter betraut ist, wie beispielsweise die Überprüfung und die Archivierung aller relevanten Prüfdokumente, das Erstellen von Maßnahmenplänen (inkl. Abstellmaßnahmen) für fehlerhafte Anlagen sowie Messungen zur Nachvermessung von eventuell nicht korrekt messender Messtechnik.

Die Integration von QS und Produktion und die damit verbundene geteilte Verantwortung von QS- und Produktions-Mitarbeitern führt zwar zu einer besseren Zusammenarbeit, der Zielkonflikt zwischen möglichst hoher produzierter Stückzahl und Qualität als oberster Maxime bleibt aber bestehen.

Herausforderung. Aufgrund der veränderten Situation ist nicht nur der Anspruch an den Umfang der Qualifikationen eines QS-Mitarbeiters gestiegen, sondern auch an die Qualifikationen eines Produktions-Mitarbeiters bzgl. Qualitätssicherung. Aufgrund des verbreiterten Aufgabenspektrums für die QS und des generell vorherrschenden Effizienzanspruchs ist es für beide Seiten eine Herausforderung, sich die benötigten Qualifikationen anzueignen. Sind die Produktions-Mitarbeiter jedoch nicht ausreichend geschult, fällt dieser Mangel in Form von zusätzlichen Unterstützungsanfragen auf die QS zurück. Daneben hat die QS als Dienstleister ein hohes Interesse daran, die Produktions-Mitarbeiter best- und schnellstmöglich in der Erledigung der qualitätsbezogenen Aufgaben zu unterstützen. Um dies zu erreichen und gleichzeitig die Anzahl ungeplanter Unterstützungseinsätze wie bspw. Abnahmen von Einstellungen bei Neuanläufen zu minimieren ist ein hohes $\mathrm{Ma} \beta$ an zielgerichteter Abstimmung zwischen Produktions- und QS-Mitarbeitern notwendig.

Lösungsansatz. Im vorliegenden Fall besteht wie oben gezeigt ein hoher Bedarf an Kompetenzerwerb und austausch auf Seiten der QS- und Produktions-Mitarbeiter. Eine IKT-gestützte Plattform ermöglicht die kollaborative Sammlung der Vielzahl notwendiger Abläufe und Aufgaben sowie der damit verbundenen Dokumententypen und bietet damit einen verbesserten Zugang zu handlungsrelevantem Wissen für beide Seiten. Daneben unterstützt eine solche Plattform die zentrale Dokumentation von Schichtübergaben und Problemlösungsprozessen und führt gleichzeitig zu einer Reduktion von erstellten Dokumenten in Papierform. Sie verbessert damit nicht nur die Problemlösungskompetenzen, sondern trägt durch die reduzierte Anzahl an Unterstützungsanfragen durch Eigenleistungen der Produktions-Mitarbeiter auch zu reduziertem Stressempfinden der QS-Mitarbeiter bei. Die gewonnene Zeit kann somit in präventive und strategische Maßnahmen zur weiteren Steigerung der Produktionsqualität investiert werden. Das kann bspw. die Analyse von fehlerhaften Fertigungsteilen sein, um durch die hierbei gewonnenen Erkenntnisse eine noch bessere Kalibrierung der Anlagen zu erreichen und den Anteil an Ausschuss noch weiter zu reduzieren. Daneben bleibt mehr Zeit für die Abarbeitung der eigentlich geplanten Haupttätigkeiten wie die Erstellung von Prozess- und Fähigkeitslandkarten. 


\section{Diskussion der Verbesserungspotentiale}

Die Nutzbarmachung der genannten Potentiale bedingt (wie in Abb. 3 zu sehen), dass Mitarbeiter geschützter und befähigter ihren Tätigkeiten nachgehen können. Auf diese Weise steigt auch die Attraktivität der Arbeitsplätze.

Im Weiteren steht die Befähigung der Mitarbeiter im Fokus. Diese sind zunehmend mit erweiterten „Abstraktions- und Problemlösungsanforderungen“ konfrontiert (Kagermann et al. 2013). Die oben eingeführten Fallbeispiele beinhalten hinsichtlich der Befähigung der Produktionsmitarbeiter weitreichende Potentiale. Für die Diskussion dieser stellt die Self-Determination Theory (SDT) (Deci et al. 1989) ein hilfreiches Rahmenwerk dar, um die Lösungen auf ihre Befähigungspotentiale hin zu untersuchen ${ }^{2}$. Die SDT unterscheidet drei grundlegende menschliche Bedürfnisse, die in Arbeits-Kontexten befriedigt werden müssen, um Wohlbefinden sowie Motivation herzustellen (Ryan und Deci 2000): Kompetenz, Verbundenheit und Autonomie. Im Kontext von Arbeitssituationen gehört zu diesen Bedürfnissen zusätzlich noch der Abwechslungsreichtum (Turner und Lawrence 1965).

\section{Bedürfnis nach ...}

(1) ... Kompetenz - der Wunsch Einfluss auf ein (Arbeits-)Ergebnis zu haben, es kontrollieren zu können.

(2) ... Verbundenheit - der Wunsch zu interagieren und verbunden zu sein.

(3) ... Autonomie - der Wunsch eine Entscheidung selbst treffen zu können.

(4) ... Abwechslungsreichtum - der Wunsch nach einem breiten Tätigkeitsspektrum.

Dabei können diese Bedürfnisse auch voneinander abhängen bzw. sich gegenseitig bedingen. Soll beispielsweise die Autonomie durch eigenverantwortlich ausgeführte, neue Tätigkeiten erhöht werden, ist in vielen Fällen auch der Aufbau von Kompetenz notwendig und der Abwechslungsreichtum erhöht sich. Im Weiteren dienen diese vier Bedürfnisse als Grundlage, um die Veränderungen in den genannten Fallbeispielen aufzuzeigen.

\subsection{Autonomie}

Im Fallbeispiel 1 (EMO) trägt die skizzierte Lösung zu einer Umstellung von ereignisgetriebener auf taktische Arbeitsplanung bei und somit auch zu mehr Autonomie. Das eingesetzte Informationssystem schafft die Datengrundlage für die selbstständige Planung der täglichen Aktivitäten. Die Auslöser für das eigene Handeln ${ }^{3}$ kommen nicht mehr von externer Seite - wie beispielsweise durch das ,plötzlich“ fehlende Teil oder die unerwartete Fertigstellung dieses Teils - sondern jetzt durch eine eigen-

\footnotetext{
${ }^{2}$ Die Bedürfnisse der Self-Determination, dt. Selbstbestimmung, sind größtenteils deckungsgleich mit dem Konzept der Befähigung (Spreitzer 1995) und den darin enthaltenen Faktoren.

${ }^{3}$ Fachlich spricht man vom ,perceived locus of causality“, also dem wahrgenommenen Ort der kausalen Ursachen für die eigenen Handlungen (Gagné und Deci 2005).
} 
verantwortlichere Planung der Aktivitäten. Im Fallbeispiel 3 (Schaeffler AG) wird die Autonomie der Mitarbeiter sowohl direkt als auch indirekt gefördert. Einerseits wirken sich der Kompetenzaufbau bei den Produktionsmitarbeitern und der damit verbundene erweiterte Handlungsspielraum direkt auf deren Autonomie aus. Andererseits entsteht durch die Reduktion von ereignisgetriebenen Arbeitsabläufen bei den QS-Mitarbeitern das Potential zur eigenverantwortlichen Planung langfristiger Aktivitäten wie beispielsweise die Analyse von Qualitätsmängeln, da sie weniger durch Hilfe-Anfragen von Produktionsmitarbeitern unterbrochen werden. Dies trägt zusätzlich auch zu mehr Qualität und Effizienz bei. Schliesslich sorgt der Kompetenzaufbau bei den QS-Mitarbeitern für zusätzliche Autonomie.

\subsection{Kompetenz}

Durch die Analyse der Fallbeispiele verstehen wir unter Kompetenz in diesem Kontext vor allem das Treffen von informierten Entscheidungen sowie die Fähigkeit, Probleme alleine oder im Team zu lösen. In allen drei Fallbeispielen lässt sich zeigen, dass die Fähigkeit informierte Entscheidungen zu fällen und Probleme zu lösen durch die zukünftigen Lösungen unterstützt wird. Während es im Fallbeispiel 1 die Entscheidungen zur Arbeitsplanung und -vorbereitung sind, die durch eine stringente Vernetzung der gesamten Belegschaft im Produktionsbereich erreicht werden sollen, wird im Fallbeispiel 2 (Hidria) direkt die fachliche Entscheidung bei der Behebung von Problemen mit den Fertigungsmaschinen durch Wissensmanagement unterstützt. Hierdurch wird die Handlungsempfehlung adressiert ,innovative Ansätze partizipativer Arbeitsgestaltung [...] [zu] fördern, die über [...] und Qualifikationsniveaus hinweg die ganze Breite der Belegschaften berücksichtigen" (Kagermann et al. 2013, S. 60). Die Handlungsempfehlung für Best-Practice-Sharing (Kagermann et al. 2013) wird auch im Fallbeispiel 3 aufgegriffen.

\subsection{Verbundenheit}

Die Verbundenheit kann sich vielseitig zeigen. Beispielsweise durch Assoziation und Mitwirkung an den Unternehmenszielen, der Förderung von Innovation sowie die gegenseitige Wahrnehmung (beispielsweise über die Aktivitäten anderer Mitarbeiter). Neben der Förderung von Autonomie bewirkt die Lösung im ersten Fallbeispiel auch eine Förderung der Verbundenheit der Mitarbeiter. Die Grenzen des eigenen „Arbeitssilos“ werden geöffnet, indem das System den Blick auf die Auswirkungen der eigenen Arbeit zulässt. Dies wäre beispielsweise die Kenntnis des Produktionsmitarbeiters, welcher Kollege gerade auf die hergestellten Teile wartet, oder umgekehrt aus Sicht der Montagemitarbeiter, wer gerade das benötigte Teil fertigt. Durch diese Art von Informationen ist somit auch eine Assoziation mit Team- oder Unternehmenszielen möglich. Somit lässt sich durch gezielten Technologieeinsatz neben einem eigenverantwortlichen auch ein partizipatives Arbeitsumfeld (Kagermann et al. 2013) gestalten. 


\subsection{Abwechslungsreichtum}

Fallbeispiel 3 zeigt auf, wie ein Informationssystem zu einem größeren Abwechslungsreichtum in der täglichen Arbeit führen kann. Durch die aktive Vernetzung der Mitarbeiter wird auch deren (vormals internalisiertes) Wissen zugänglich und dadurch der Tätigkeitsspielraum der Mitarbeiter erweitert. Auf der anderen Seite werden die QS-Mitarbeiter - die heute über ein großes „Inselwissen“ verfügen - entlastet, wodurch bei diesen ein größerer Planungsspielraum entsteht bzw. mehr Zeit für weitere Tätigkeiten bleibt, wie präventive und strategische Maßnahmen zur weiteren Steigerung der Produktionsqualität.

Schließlich zeigt sich an diesem Beispiel der enge Zusammenhang zwischen den einzelnen Bedürfnissen. Denn durch den o. g. Kompetenzgewinn wird gleichzeitig auch die Autonomie der Produktions-Mitarbeiter unterstützt, da sich damit auch ihr Handlungs- und Entscheidungsspielraum vergrößert.

\section{Attraktive Arbeitsplätze in der Fabrik der Zukunft}

Mit der schrittweisen Realisierung von Smart Factories werden Produktionsstätten neu gedacht und Produktionsarbeit erfährt einen wirtschaftlichen und gesellschaftlichen Wertewandel. Produktionsstandorte können somit nicht nur technologisch und wirtschaftlich, sondern auch auf der sozialen Ebene stabilisiert werden. Es existiert eine große Anzahl an Fertigungsformen, die je nach Unternehmen, Produkt und Stückzahl spezifisch den Anforderungen angepasst sind. Wie die drei im Beitrag vorgestellten Fallbeispiele zeigen, führt dies zu vielfältigen Voraussetzungen und Ausprägungen. Unternehmen mit sehr flexibel ausgerichteter Fertigung und oftmals mit geringerer Automatisierung stehen hochautomatisierten Produktionsstätten gegenüber. Beiden gemeinsam ist der Bedarf, attraktive Arbeitsplätz zu schaffen, bei denen der Mensch im Mittelpunkt steht. Trotz der unterschiedlichen Organisationen der Produktion bietet Industrie 4.0 einen gemeinsamen Schirm für das Bestreben, den Produktionsstandort in Europa durch ein Forcieren des Einsatzes von IKT zu festigen und für eine erfolgreiche Zukunft vorzubereiten. Gerade aufgrund der variierenden Anwendungsfortschritte und Anwenderniveaus bietet sich kein einheitliches Bild in der Definition des Begriffs und der Abgrenzung. Durch die Vorstellung von drei anschaulichen Fallbeispielen möchte diese Veröffentlichung dazu einen Beitrag leisten.

Neben den angestrebten organisatorischen Veränderungen leiten sich durch die Einführung von Lösungen darüber hinaus auch Entwicklungspotentiale hin zu einem Mensch-zentrierten Arbeitsplatz ab. Als Basis zur Zielerreichung werden hierbei unter anderem ,[...] die Verfügbarkeit aller relevanten Informationen in Echtzeit durch Vernetzung aller an der Wertschöpfung beteiligten Instanzen sowie die Fähigkeit aus den Daten den zu jedem Zeitpunkt optimalen Wertschöpfungsfluss abzuleiten [...]" genannt (vgl. Lucke et al. 2014).

Die drei Fallbeispiele haben gezeigt, wie ein Mitarbeiter durch den zielgerichteten Einsatz von IKT insbesondere 1) Kompetenz, 2) Verbundenheit, 3) Autonomie und 4) Abwechslungsreichtum gewinnen und damit zu einem befähigteren Mitarbeiter wachsen kann. Diese erste Diskussion der Potentiale und ihre systematische Ein- 
ordnung in ein wissenschaftliches Rahmenwerk sollen dem Leser ein umfassenderes Verständnis ermöglichen. Gleichzeitig ist davon auszugehen, dass in den kommenden Jahren noch eine Vielzahl weiterer Fallbeispiele folgen wird, die zu einem ausdifferenzierteren Verständnis der Potentiale aber auch der Grenzen von Industrie 4.0 beitragen können.

Danksagung This study has been funded by the European Commission within the FACTS 4WORKERS under the Horizon 2020 program of the European Commission within the Factories of the Future PPP.

Wolfgang Unzeitig and Alexander Stocker acknowledge the financial support of the COMET K2Competence Centres for Excellent Technologies Programme of the Austrian Federal Ministry for Transport, Innovation and Technology (BMVIT), the Austrian Federal Ministry of Science, Research and Economy (BMWFW), the Austrian Research Promotion Agency (FFG), the Province of Styria and the Styrian Business Promotion Agency (SFG).

\section{Anhang}

Tab. 1 Überblick über die analysierten Projekte im Rahmen von Factories of the Future, die den Themengebieten „Collaborative and Mobile Enterprises“ und „Human Centred Manufacturing“ zugeordnet werden

\begin{tabular}{|c|c|c|}
\hline Projekt & FoF Thema & Vollständiger Projektname \\
\hline VENIS & $\begin{array}{l}\text { Collaborative and } \\
\text { Mobile Enterprises }\end{array}$ & Virtual Enterprises by Networked Interoperability Services \\
\hline MEMAN & $\begin{array}{l}\text { Collaborative and } \\
\text { Mobile Enterprises }\end{array}$ & $\begin{array}{l}\text { Integral Material and Energy flow MANagement in MANu- } \\
\text { facturing metal mechanic sector }\end{array}$ \\
\hline C2NET & $\begin{array}{l}\text { Collaborative and } \\
\text { Mobile Enterprises }\end{array}$ & Cloud Collaborative Manufacturing Networks \\
\hline CREMA & $\begin{array}{l}\text { Collaborative and } \\
\text { Mobile Enterprises }\end{array}$ & Cloud-based Rapid Elastic MAnufacturing \\
\hline MANSYS & $\begin{array}{l}\text { Collaborative and } \\
\text { Mobile Enterprises }\end{array}$ & $\begin{array}{l}\text { MANufacturing decision and supply chain management } \\
\text { SYStem for additive manufacturing }\end{array}$ \\
\hline ROBUSTPLANET & $\begin{array}{l}\text { Collaborative and } \\
\text { Mobile Enterprises }\end{array}$ & $\begin{array}{l}\text { Shock-robust Design of Plants and their Supply Chain } \\
\text { Networks }\end{array}$ \\
\hline FLEXINET & $\begin{array}{l}\text { Collaborative and } \\
\text { Mobile Enterprises }\end{array}$ & $\begin{array}{l}\text { Intelligent Systems Configuration Services for Flexible } \\
\text { Dynamic Global Production Networks }\end{array}$ \\
\hline USE-IT-WISELY & $\begin{array}{l}\text { Collaborative and } \\
\text { Mobile Enterprises }\end{array}$ & $\begin{array}{l}\text { Innovative continuous upgrades of high investment } \\
\text { product-services }\end{array}$ \\
\hline EASY-IMP & $\begin{array}{l}\text { Collaborative and } \\
\text { Mobile Enterprises }\end{array}$ & $\begin{array}{l}\text { Collaborative Development of Intelligent Wearable Meta- } \\
\text { Products in the Cloud }\end{array}$ \\
\hline PROSECO & $\begin{array}{l}\text { Collaborative and } \\
\text { Mobile Enterprises }\end{array}$ & $\begin{array}{l}\text { Collaborative Environment for Design of AmI enhanced } \\
\text { Product-Services Integrating Highly Personalised Innovati- } \\
\text { ve Functions with Minimal Ecological Footprint along Life } \\
\text { Cycle and of Their Production }\end{array}$ \\
\hline ROAD4FAME & $\begin{array}{l}\text { Collaborative and } \\
\text { Mobile Enterprises }\end{array}$ & $\begin{array}{l}\text { Development of a Strategic Research and Innovation Road- } \\
\text { map for Future Architectures and Services for Manufactu- } \\
\text { ring in Europe and Derivation of Business Opportunities }\end{array}$ \\
\hline FOFDATION & $\begin{array}{l}\text { Collaborative and } \\
\text { Mobile Enterprises }\end{array}$ & The Foundation for the Smart Factory of the Future \\
\hline ADVENTURE & $\begin{array}{l}\text { Collaborative and } \\
\text { Mobile Enterprises }\end{array}$ & ADaptive Virtual ENterprise ManufacTURing Environmen \\
\hline
\end{tabular}


Tab. 1 (Fortsetzung)

\begin{tabular}{|c|c|c|}
\hline Projekt & FoF Thema & Vollständiger Projektname \\
\hline BIVEE & $\begin{array}{l}\text { Collaborative and } \\
\text { Mobile Enterprises }\end{array}$ & Business Innovation and Virtual Enterprise Environment \\
\hline COMVANTAGE & $\begin{array}{l}\text { Collaborative and } \\
\text { Mobile Enterprises }\end{array}$ & $\begin{array}{l}\text { Collaborative Manufacturing Network for Competitive } \\
\text { Advantage }\end{array}$ \\
\hline EPES & $\begin{array}{l}\text { Collaborative and } \\
\text { Mobile Enterprises }\end{array}$ & $\begin{array}{l}\text { ECO-PROCESS ENGINEERING SYSTEM FOR COM- } \\
\text { POSITION OF SERVICES TO OPTIMIZE PRODUCT } \\
\text { LIFE-CYCLE }\end{array}$ \\
\hline $\begin{array}{l}\text { EXTREMEFAC- } \\
\text { TORIES }\end{array}$ & $\begin{array}{l}\text { Collaborative and } \\
\text { Mobile Enterprises }\end{array}$ & $\begin{array}{l}\text { On-the-cloud environment implementing agile management } \\
\text { methods for enabling the set-up, monitoring and follow-up } \\
\text { of business innovation processes in industrial SMEs }\end{array}$ \\
\hline GLONET & $\begin{array}{l}\text { Collaborative and } \\
\text { Mobile Enterprises }\end{array}$ & $\begin{array}{l}\text { Glocal enterprise network focusing on customer-centric } \\
\text { collaboration }\end{array}$ \\
\hline IMAGINE & $\begin{array}{l}\text { Collaborative and } \\
\text { Mobile Enterprises }\end{array}$ & $\begin{array}{l}\text { Innovative End-to-end Management of Dynamic Manufac- } \\
\text { turing Networks }\end{array}$ \\
\hline MSEE & $\begin{array}{l}\text { Collaborative and } \\
\text { Mobile Enterprises }\end{array}$ & Manufacturing SErvice Ecosystem \\
\hline PREMANUS & $\begin{array}{l}\text { Collaborative and } \\
\text { Mobile Enterprises }\end{array}$ & Product Remanufacturing Service System \\
\hline MAN-MADE & $\begin{array}{l}\text { Human Centred } \\
\text { Manufacturing }\end{array}$ & $\begin{array}{l}\text { MANufacturing through ergonoMic and safe Anthropo- } \\
\text { centric aDaptive workplacEs for context aware factories in } \\
\text { EUROPE }\end{array}$ \\
\hline SO-PC-Pro & $\begin{array}{l}\text { Human Centred } \\
\text { Manufacturing }\end{array}$ & Subject Orientation For People Centred Production \\
\hline SO SMART & $\begin{array}{l}\text { Human Centred } \\
\text { Manufacturing }\end{array}$ & $\begin{array}{l}\text { Socially sustainable manufacturing for the Factories of the } \\
\text { Future }\end{array}$ \\
\hline $\begin{array}{l}\text { FACTS } \\
\text { 4WORKERS }\end{array}$ & $\begin{array}{l}\text { Human Centred } \\
\text { Manufacturing }\end{array}$ & Worker-Centric Workplaces in Smart Factories \\
\hline SATISFACTORY & $\begin{array}{l}\text { Human Centred } \\
\text { Manufacturing }\end{array}$ & $\begin{array}{l}\text { A collaborative and augmented-enabled ecosystem for } \\
\text { increasing SATISfaction and working experience in smart } \\
\text { FACTORY environments }\end{array}$ \\
\hline LIAA & $\begin{array}{l}\text { Human Centred } \\
\text { Manufacturing }\end{array}$ & Lean Intelligent Assembly Automation \\
\hline ROBO-PARTNER & $\begin{array}{l}\text { Human Centred } \\
\text { Manufacturing }\end{array}$ & $\begin{array}{l}\text { Seamless Human-Robot Cooperation for Intelligent, } \\
\text { Flexible and Safe Operations in the Assembly Factories of } \\
\text { the Future }\end{array}$ \\
\hline ROBO-MATE & $\begin{array}{l}\text { Human Centred } \\
\text { Manufacturing }\end{array}$ & $\begin{array}{l}\text { Intelligent exoskeleton based on human-robot interaction } \\
\text { for manipulation of heavy goods in Europe's factories of } \\
\text { the future }\end{array}$ \\
\hline $\begin{array}{l}\text { FACTORY-IN- } \\
\text { A-DAY }\end{array}$ & $\begin{array}{l}\text { Human Centred } \\
\text { Manufacturing }\end{array}$ & Factory-in-a-day \\
\hline FOURBYTHREE & $\begin{array}{l}\text { Human Centred } \\
\text { Manufacturing }\end{array}$ & $\begin{array}{l}\text { Highly customizable robotic solutions for effective and safe } \\
\text { human robot collaboration in manufacturing applications }\end{array}$ \\
\hline SENSE\&REACT & $\begin{array}{l}\text { Human Centred } \\
\text { Manufacturing }\end{array}$ & $\begin{array}{l}\text { Sense\&React - The context-aware and user-centric infor- } \\
\text { mation distribution system for manufacturing }\end{array}$ \\
\hline KNOW-4-CAR & $\begin{array}{l}\text { Human Centred } \\
\text { Manufacturing }\end{array}$ & $\begin{array}{l}\text { An Internet-based Collaborative Platform for Managing } \\
\text { Manufacturing Knowledge }\end{array}$ \\
\hline MANUSKILLS & $\begin{array}{l}\text { Human Centred } \\
\text { Manufacturing }\end{array}$ & $\begin{array}{l}\text { Envisioning an advanced ICT-supported build-up of manu- } \\
\text { facturing skills for the Factories of the Future }\end{array}$ \\
\hline VISTRA & $\begin{array}{l}\text { Human Centred } \\
\text { Manufacturing }\end{array}$ & $\begin{array}{l}\text { Virtual Simulation and Training of Assembly and Service } \\
\text { Processes in Digital Factories }\end{array}$ \\
\hline
\end{tabular}


A. Richter et al.

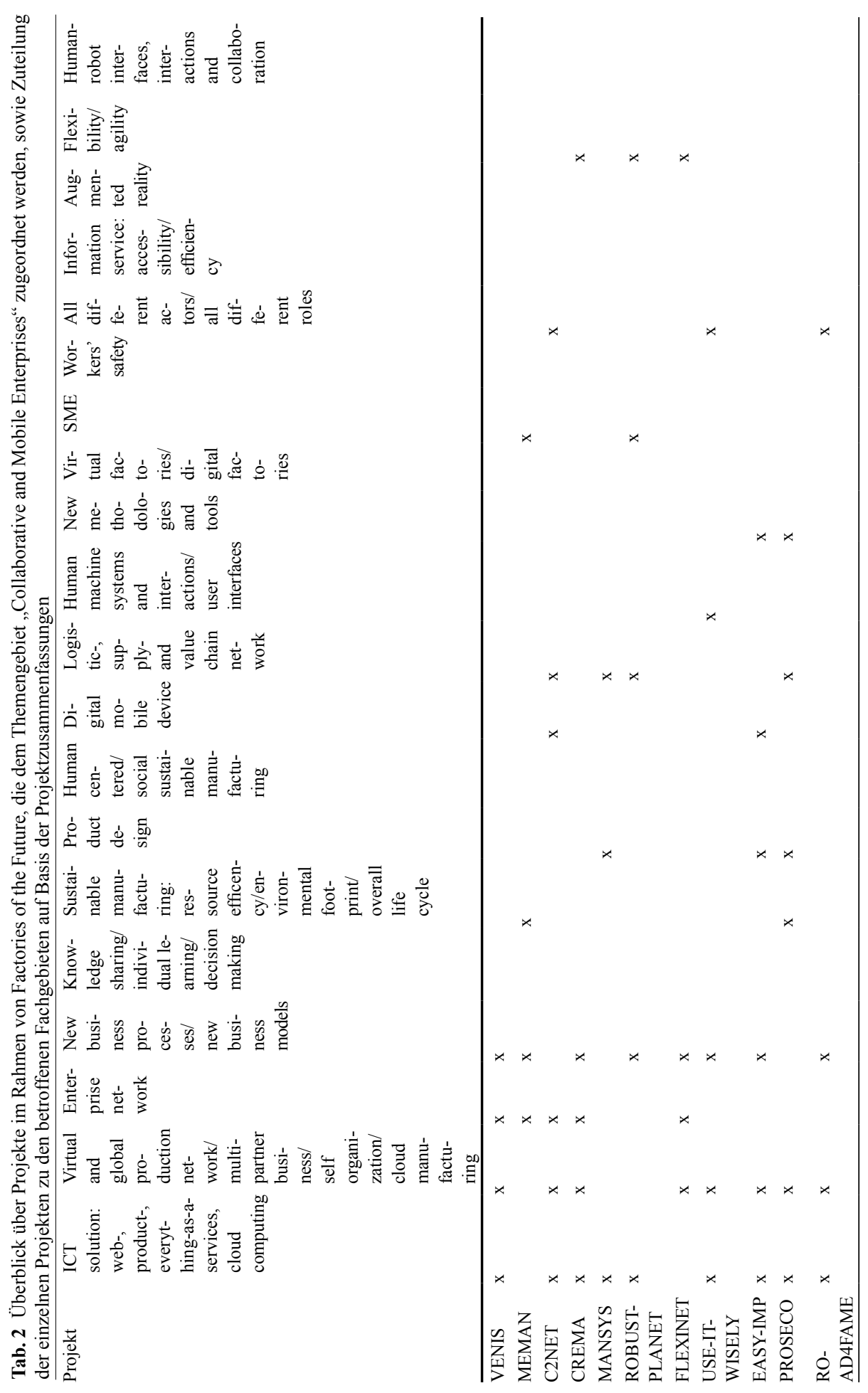




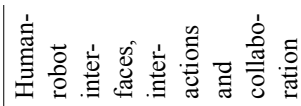

这兽莺

完离害窟

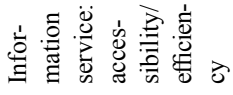

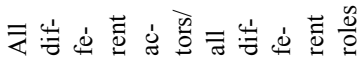

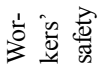

$\sum_{i=1}^{\infty}$

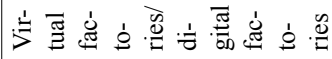

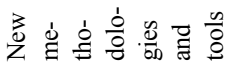

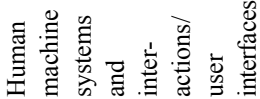

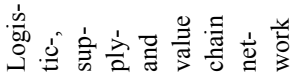

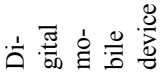

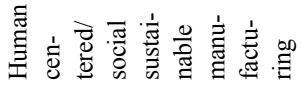

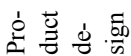

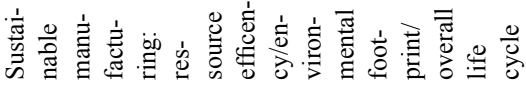

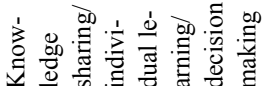

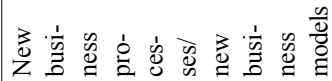

离点离薏

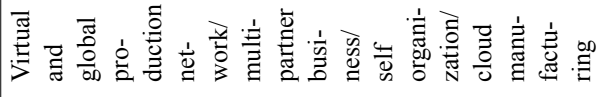

Oొ

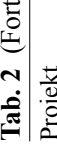

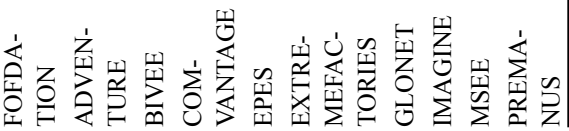


A. Richter et al.

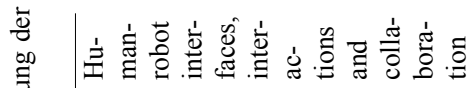

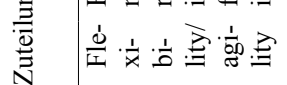

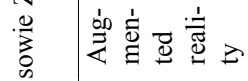

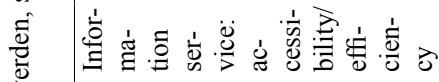

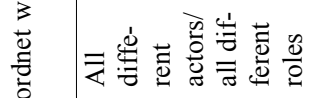

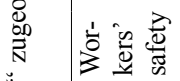

兽

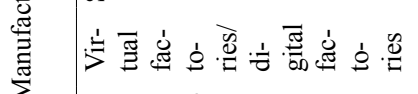

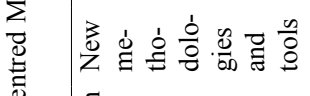

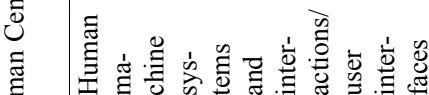

焉

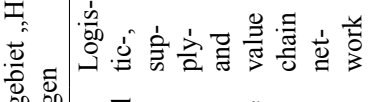

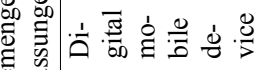

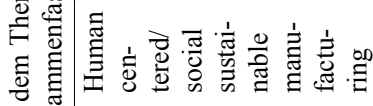

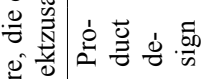

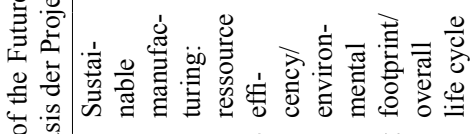

怘

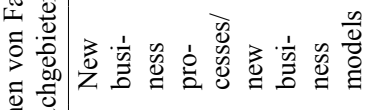

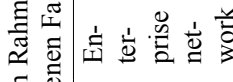

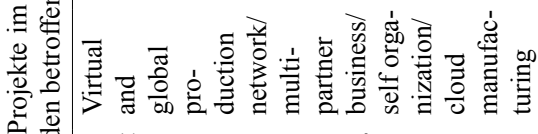

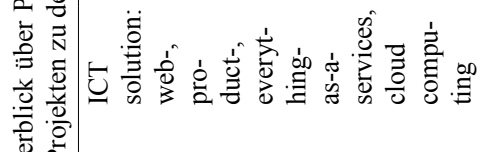

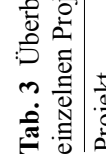

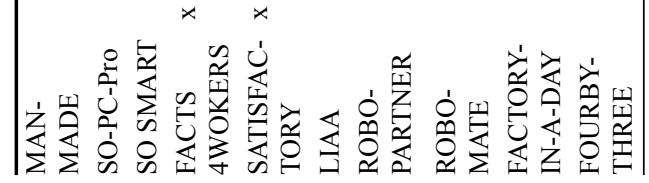

iㅡㄹ Springer 


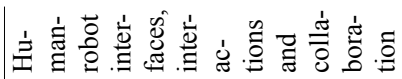

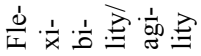

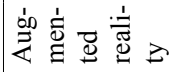

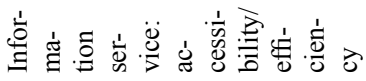

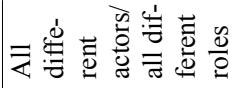

它的密

$\sum_{i=1}^{1}$

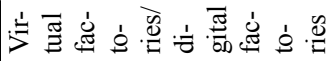

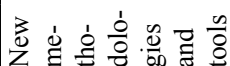

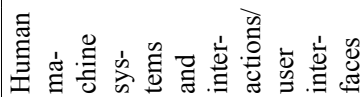

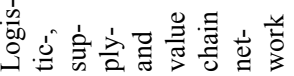

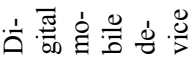

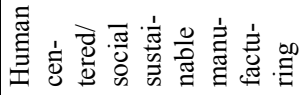

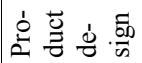

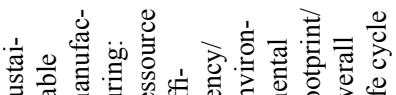

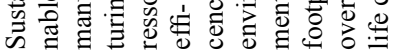

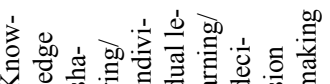

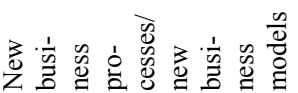

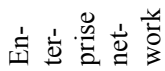

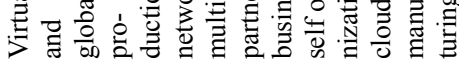

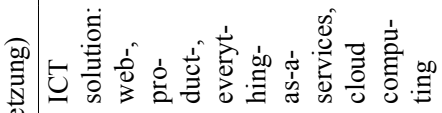


Tab. 4 Adressierte Themengebiete in den FOF-Projekten im Bereich „Collaborative and Mobile Enterprises“ und „Human Centred Manufacturing“ und Zuordnung zum IoT Technology Stack, ergänzt um Manufacturing und Engineering (Wortmann und Flüchter 2015, nach Porter und Heppelmann 2014)

\begin{tabular}{|c|c|c|c|}
\hline $\begin{array}{l}\text { Kategorie nach Wort- } \\
\text { mann \& Flüchter: } \\
\text { Feinkategorie } \\
\text { (Grobkategorie) }\end{array}$ & $\begin{array}{l}\text { Themengebiete der FoF } \\
\text { Projekte }\end{array}$ & Erklärung des Themas & Beispielprojekte \\
\hline \multirow[t]{5}{*}{$\begin{array}{l}\text { IoT applications (IoT } \\
\text { Cloud) }\end{array}$} & $\begin{array}{l}\text { ICT solution: } \\
\text { web-, product-, } \\
\text { everything-as-a-services }\end{array}$ & $\begin{array}{l}\text { Dienstleistungen, die } \\
\text { einerseits über das Internet } \\
\text { und die andererseits zu- } \\
\text { sätzlich zum produzierten } \\
\text { Gut beziehungsweise die } \\
\text { als der Herstellungsprozess } \\
\text { an sich zur Verfügung ge- } \\
\text { stellt werden }\end{array}$ & $\begin{array}{l}\text { VENIS, C2NET, } \\
\text { CREMA }\end{array}$ \\
\hline & $\begin{array}{l}\text { All different actors/all } \\
\text { different roles }\end{array}$ & $\begin{array}{l}\text { Berücksichtigung von } \\
\text { allen verschiedenen Rollen } \\
\text { und Akteuren im Unter- } \\
\text { nehmen vom Produkions- } \\
\text { arbeiter bis zum Manager }\end{array}$ & $\begin{array}{l}\text { USE-IT-WISE- } \\
\text { LY, ROAD4FA- } \\
\text { ME, FACTS } \\
\text { 4WORKERS }\end{array}$ \\
\hline & $\begin{array}{l}\text { Human machine systems } \\
\text { and interactions/user } \\
\text { interfaces }\end{array}$ & $\begin{array}{l}\text { Systeme von Mensch } \\
\text { und Maschinen mit } \\
\text { entsprechenden } \\
\text { Anwenderschnittstellen }\end{array}$ & $\begin{array}{l}\text { SO-PC-Pro, SO } \\
\text { SMART, FACTS } \\
\text { 4WORKERS }\end{array}$ \\
\hline & $\begin{array}{l}\text { Information service: } \\
\text { accessibility/efficiency }\end{array}$ & $\begin{array}{l}\text { Effizientes und zugäng- } \\
\text { liches Bereitstellen von } \\
\text { Informationen }\end{array}$ & $\begin{array}{l}\text { SENSE\&REACT, } \\
\text { ROBO-PART- } \\
\text { NER, FACTS } \\
\text { 4WORKERS }\end{array}$ \\
\hline & $\begin{array}{l}\text { Human-robot inter- } \\
\text { faces, interactions and } \\
\text { collaboration }\end{array}$ & $\begin{array}{l}\text { Schnittstellen zwischen } \\
\text { Mensch und Roboter mit } \\
\text { dem Ziel einer Interaktion } \\
\text { und Kollaboration von } \\
\text { beiden }\end{array}$ & $\begin{array}{l}\text { ROBO-PARTNER, } \\
\text { ROBO-MATE, } \\
\text { FACTORY-IN-A- } \\
\text { DAY }\end{array}$ \\
\hline \multirow[t]{2}{*}{$\begin{array}{l}\text { Analytics and data ma- } \\
\text { nagement (IoT Cloud) }\end{array}$} & $\begin{array}{l}\text { Knowledge sharing/indi- } \\
\text { vidual learning/decision } \\
\text { making }\end{array}$ & $\begin{array}{l}\text { Teilen von Wissen und } \\
\text { Unterstützung von } \\
\text { individuellem Lernen als } \\
\text { Grundlage für unterneh- } \\
\text { mensspezifische Entschei- } \\
\text { dungen vom einzelnen }\end{array}$ & $\begin{array}{l}\text { KNOW-4- } \\
\text { CAR, MAN- } \\
\text { MADE, FACTS } \\
\text { 4WORKERS }\end{array}$ \\
\hline & Augmented reality & $\begin{array}{l}\text { Virtuelle Erweiterung der } \\
\text { realen Daten }\end{array}$ & $\begin{array}{l}\text { SATISFACTORY, } \\
\text { LIAA, FACTS } \\
\text { 4WORKERS }\end{array}$ \\
\hline $\begin{array}{l}\text { Process management } \\
\text { (IoT Cloud) }\end{array}$ & $\begin{array}{l}\text { New business processes/ } \\
\text { new business models }\end{array}$ & $\begin{array}{l}\text { Neue Unterneh- } \\
\text { mensprozesse und } \\
\text { Geschäftsmodelle }\end{array}$ & $\begin{array}{l}\text { ROBUSTPLA- } \\
\text { NET, FLEXINET, } \\
\text { COMVANTAGE }\end{array}$ \\
\hline \multirow[t]{2}{*}{$\begin{array}{l}\text { Network communica- } \\
\text { tion (Connectivity) }\end{array}$} & Cloud computing & $\begin{array}{l}\text { Verwendung von ent- } \\
\text { fernten Rechenzentren zur } \\
\text { Informationsverarbeitung }\end{array}$ & $\begin{array}{l}\text { CREMA, EASY- } \\
\text { IMP, C2NET }\end{array}$ \\
\hline & $\begin{array}{l}\text { Virtual and global } \\
\text { production network/ } \\
\text { multi-partner business/ } \\
\text { self organization/cloud } \\
\text { manufacturing }\end{array}$ & $\begin{array}{l}\text { Virtuelles und globales } \\
\text { Unternehmensnetzwerk } \\
\text { mit mehrerern Unter- } \\
\text { nehmenspartnern, die } \\
\text { sich automatisiert selbst } \\
\text { unterstützen und aus einer } \\
\text { Produktions-Cloud abgeru- } \\
\text { fen werden können }\end{array}$ & $\begin{array}{l}\text { FLEXINET, } \\
\text { PROSECO, } \\
\text { FOFDATION }\end{array}$ \\
\hline
\end{tabular}


Tab. 4 (Fortsetzung)

\begin{tabular}{|c|c|c|c|}
\hline $\begin{array}{l}\text { Kategorie nach Wort- } \\
\text { mann \& Flüchter: } \\
\text { Feinkategorie } \\
\text { (Grobkategorie) }\end{array}$ & $\begin{array}{l}\text { Themengebiete der FoF } \\
\text { Projekte }\end{array}$ & Erklärung des Themas & Beispielprojekte \\
\hline & Enterprise network & Unternehmensnetzwerk & $\begin{array}{l}\text { ADVENTU- } \\
\text { RE, BIVEE, } \\
\text { COMVANTAGE }\end{array}$ \\
\hline & $\begin{array}{l}\text { Logistic-, supply- and } \\
\text { value chain network }\end{array}$ & $\begin{array}{l}\text { Logistik-, Zuliefer-, } \\
\text { Wertschöfpungskette }\end{array}$ & $\begin{array}{l}\text { MANSYS, RO- } \\
\text { BUSTPLANET, } \\
\text { IMAGINE }\end{array}$ \\
\hline & Digital mobile device & $\begin{array}{l}\text { Verwendung von } \\
\text { Hardware-Komponenten }\end{array}$ & $\begin{array}{l}\text { C2NET, EASY- } \\
\text { IMP, FACTS } \\
\text { 4WORKERS }\end{array}$ \\
\hline \multirow[t]{7}{*}{ Manufacturing } & $\begin{array}{l}\text { Sustainable manu- } \\
\text { facturing: ressource } \\
\text { efficency/environmental } \\
\text { footprint/overall life } \\
\text { cycle }\end{array}$ & $\begin{array}{l}\text { Nachhaltige Produktion } \\
\text { hinsichtlich Ressourcen- } \\
\text { effizienz, ökologischen } \\
\text { Fußabdruck über den } \\
\text { gesamten Lebenszyklus } \\
\text { hinweg }\end{array}$ & $\begin{array}{l}\text { MEMAN, EPES, } \\
\text { PREMANUS }\end{array}$ \\
\hline & $\begin{array}{l}\text { Human centered/ } \\
\text { social sustainable } \\
\text { manufacturing }\end{array}$ & $\begin{array}{l}\text { Sozial nachhaltige Produk- } \\
\text { tion mit dem Menschen im } \\
\text { Mittelpunkt }\end{array}$ & $\begin{array}{l}\text { MAN-MADE, } \\
\text { SATISFAC- } \\
\text { TORY, FACTS } \\
\text { 4WORKERS }\end{array}$ \\
\hline & $\begin{array}{l}\text { New methodologies and } \\
\text { tools }\end{array}$ & $\begin{array}{l}\text { Entwicklung neuer Metho- } \\
\text { den und Werkzeuge }\end{array}$ & $\begin{array}{l}\text { EASY-IMP, PROSE- } \\
\text { CO, EPES }\end{array}$ \\
\hline & $\begin{array}{l}\text { Virtual factories/digital } \\
\text { factories }\end{array}$ & $\begin{array}{l}\text { Digitale Fabrik als virtuel- } \\
\text { le Abbild der realen Fabrik } \\
\text { zur ganzheitlichen Planung } \\
\text { und Verbesserung dieser } \\
\text { (VDI4499) }\end{array}$ & $\begin{array}{l}\text { ADVENTU- } \\
\text { RE, BIVEE, } \\
\text { COMVANTAGE }\end{array}$ \\
\hline & SME & $\begin{array}{l}\text { Besonderer Fokus auf } \\
\text { kleine und mittlere } \\
\text { Unternehmen }\end{array}$ & $\begin{array}{l}\text { ADVENTURE, } \\
\text { COMVANTAGE, } \\
\text { EXTREMEFACTO- } \\
\text { RIES }\end{array}$ \\
\hline & Workers' safety & Arbeitssicherheit & $\begin{array}{l}\text { ROBO-PARTNER, } \\
\text { ROBO-MATE, } \\
\text { FACTORY-IN-A- } \\
\text { DAY }\end{array}$ \\
\hline & Flexibility/agility & $\begin{array}{l}\text { Flexible und agile Aus- } \\
\text { legung des Produktion und } \\
\text { des Produktionssystems }\end{array}$ & $\begin{array}{l}\text { CREMA, FLEXI- } \\
\text { NET, ADVENTURE }\end{array}$ \\
\hline Engineering & Product design & $\begin{array}{l}\text { Integration der } \\
\text { Produktentwicklung }\end{array}$ & $\begin{array}{l}\text { MANSYS, EPES, } \\
\text { IMAGINE }\end{array}$ \\
\hline
\end{tabular}




\section{Literatur}

Deci EL, Connell JP, Ryan MR (1989) Self-determination in a work organization. J Appl Psychol 74(4):580 Denger A, Fritz J, Denger D, Priller P, Kaiser C, Stocker A (2014) Organisationaler Wandel durch die Emergenz Cyber-Physikalischer Systeme: Die Fallstudie AVL List GmbH. HMD Prax Wirtsch 51(6):827-837

EFFRA (2013) Factories of the future 2020' roadmap 2014-2020. http://www.effra.eu/attachments/ article/129/Factories\%20of\%20the\%20Future\%202020\%20Roadmap.pdf. Zugegriffen: 25 . Juni 2015

European Commission (2015) Public private partnerships in research, factories of the future. http://ec.europa.eu/research/industrial_technologies/factories-of-the-future_en.html. Zugegriffen: 25. Juni 2015

Gagné M, Deci EL (2005) Self-determination theory and work motivation. J Organ Behav 26(4):331-362

Kagermann H (2014). Der Mitarbeiter selbst wird wieder im Vordergrund stehen. http://www.zeit.de/karriere/beruf/2014-11/henning-kagermann-zukunft-arbeit-interview. Zugegriffen: 24. Juni 2015

Kagermann H, Wahlster W, Helbig J (2013) Umsetzungsempfehlungen für das Zukunftsprojekt Industrie 4.0: Abschlussbericht des Arbeitskreises Industrie 4.0, acatech-National Academy of Science and Engineering. Munich

Lucke D, Görzig D, Kacir M, Volkmann J, Haist C, Sachsenmaier M, Rentschler H (2014) Strukturstudie Industrie 4.0 für Baden-Württemberg." 2014. Fraunhofer-Institut für Produktionstechnik und Automatisierung IPA. http://mfw.baden-wuerttemberg.de/fileadmin/redaktion/m-mfw/intern/Dateien/ Downloads/Industrie_und_Innovation/IPA_Strukturstudie_Industrie_4.0_BW.pdf. Zugegriffen: 24. Juni 2015

Porter ME, Heppelmann JE (2014) How smart, connected products are transforming competition, Harvard Business Review, November 2014

Ryan RM, Deci EL (2000) Self-determination theory and the facilitation of intrinsic motivation, social development, and well-being. Am Psychol 55(1):68

Spath D, Ganschar O, Gerlach S, Hämmerle M, Krause T, Schlund S (2013) Produktionsarbeit der Zukunft - Industrie 4.0, Fraunhofer Institut für Arbeitswissenschaft und Organisation IAO. Fraunhofer, Stuttgart

Spreitzer GM (1995) Psychological empowerment in the workplace: dimensions, measurement, and validation. Acad Manag J 38(5):1442-1465. doi:10.2307/256865

Turner AN, Lawrence PR (1965) Industrial jobs and the workers: an investigation of response to task attributes. Harvard University, Division of Research, Graduate School of Business Administration, Boston

Unzeitig W, Rubesa J, Schafler M, Stocker A, Ramsauer C, Flasch M (2014) Eine Methode zur Berücksichtigung von Unsicherheit im Zuge der frühen Fabrikplanung in der Auftragsfertigung von Gesamtfahrzeugen, e \& i Elektrotechnik und Informationstechnik, Vol. 131, Is. 7, Oktober 2014

Wortmann F, Flüchter K (2015) Internet of things: technology and value added. Bus Inf Syst Eng 57(3):221-224 